\begin{tabular}{|c|c|}
\hline Title & Light propagation model of titanium dioxide suspensions in water using the radiative transfer equation \\
\hline Author(s) & Fujii, Hiroy uki; Y amada, Y ukio; Hoshi, Y oko; Okawa, Shinpei; Kobay ashi, Kazumichi; W atanabe, Masao \\
\hline Citation & $\begin{array}{l}\text { Reaction kinetics, mechanisms and catalysis, 123(2), } 439-453 \\
\text { https://doi.org/10.1007/s11144 017-1328-2 }\end{array}$ \\
\hline Issue Date & $2018-04$ \\
\hline Doc URL & http:/hdl.handle.net/2115/73357 \\
\hline Rights & $\begin{array}{l}\text { This is a post-peer-review, pre copy edit version of an article published in Reaction Kinetics, Mechanisms and Catalysis. } \\
\text { The final authenticated version is available online at: } h(t+p: / / d x . d o i . o r g / 10.1007 / 511144-017-1328-2 \text {. }\end{array}$ \\
\hline Type & article (author version) \\
\hline File Information & RKMC_Fujii.pdf \\
\hline
\end{tabular}

Instructions for use 


\title{
Light propagation model of titanium dioxide suspensions in water using the radiative transfer equation
}

\author{
Hiroyuki Fujii · Yukio Yamada · Yoko \\ Hoshi · Shinpei Okawa · Kazumichi \\ Kobayashi · Masao Watanabe
}

Received: date / Accepted: date

\begin{abstract}
Constructions of numerical schemes for solving the radiative transfer equation (RTE) are crucial to evaluate light propagation inside photocatalytic systems. We develop accurate and efficient schemes of the three-dimensional and time-dependent RTE for numerical phantoms modeling aqueous titanium dioxide suspensions, in which the anisotropy of the forward-directed scattering varies and the strength of absorption is comparable to that of scattering. To improve the accuracy and efficiency of the numerical solutions, the forward-directed phase function is renormalized in the zeroth or first order with a small number of discrete angular directions. Then, we investigate the influences of the forward-directed scattering on the numerical solutions by comparing with the analytical solutions. The investigation shows that with the anisotropy factor less than approximately 0.7 corresponding to the moderate forward-directed scattering, the numerical solutions of the RTE using the both of the zeroth and first order renormalization approaches are accurate due to the reductions of the numerical errors of the phase function. With the anisotropy factor more than approximately 0.7 corresponding
\end{abstract}

\section{H. Fujii}

Division of Mechanical and Space Engineering, Faculty of Engineering, Hokkaido University, Kita 13 Nishi 8, Kita-ku, Sapporo, Hokkaido 060-8628, Japan

E-mail: fujii-hr@eng.hokudai.ac.jp

\section{Y. Yamada}

Brain Science Inspired Life Support Research Center, University of Electro-Communications, 1-5-1 Chofugaoka, Chofu, Tokyo 182-8585, Japan

Y. Hoshi

Preeminent Medical Photonics Education \& Reseach Center, Hamamatsu University School of Medicine, 1-20-1 Handayama, Higashi-ku, Hamamatsu, Sizuoka 431-3192, Japan

S. Okawa

National Defense Medical College, 3-2 Namiki, Tokorozawa, Saitama, 359-8513 Japan

K. Kobayashi

Division of Mechanical and Space Engineering, Faculty of Engineering, Hokkaido University, Kita 13 Nishi 8, Kita-ku, Sapporo, Hokkaido 060-8628, Japan

M. Watanabe

Division of Mechanical and Space Engineering, Faculty of Engineering, Hokkaido University, Kita 13 Nishi 8, Kita-ku, Sapporo, Hokkaido 060-8628, Japan 
to the highly forward-directed scattering, the first order renormalization approach still provides the accurate results, while the zeroth order approach does not due to the large errors of the phase function. These results suggest that the developed scheme using the first order renormalization can provide accurate and efficient calculations of light propagation in photocatalytic systems.

Keywords Light propagation in photocatalytic systems · radiative transfer equation - renormalization approach of the forward-directed phase function

Mathematics Subject Classification (2000) 78A40 - 92E20 - 65D30 - 74E10

\section{Introduction}

Evaluation of light propagation inside the photocatalytic reactors is crucial for a reactor design, kinetic modeling in the reactors $[1,2]$, and photocatalytic performance evaluation. The radiative transfer equation (RTE) or linear Boltzmann equation can accurately describe light propagation inside turbid media such as photocatalytic systems $[3,4,5]$. For calculations of the RTE, one needs the optical properties (absorption and scattering coefficients; and anisotropy factor) as parameters. Absorption and scattering coefficients represent the strengths of light absorption and scattering, respectively. The anisotropy factor ranging from -1 to 1 characterizes the anisotropy of light scattering. The zero value of the factor corresponds to isotropic scattering, the value of -1 to purely backward-directed scattering, and the value of 1 to purely forward-directed scattering. Excellent studies have determined the optical properties of the photocatalytic systems $[6,3,7]$ by using the RTE. The researches show that most of photocatalytic systems are highly forward-directed scattering media, e.g. the anisotropy factor of silica supported titanium dioxide suspensions is in a range from 0.75 to 0.88 [3].

Usually, the RTE is solved numerically because the photocatalytic reactors are heterogeneous media, for which it is quite difficult to derive the analytical solutions of the RTE. For angular discretization of the RTE, the discrete ordinates methods (DOM) have been widely used with a quadrature set $[8,9]$. The highly forward-directed scattering such as photocatalytic systems requires a large number of discrete angular directions to obtain accurate solutions of the RTE. This fact leads to high computational costs. For example, the previous studies $[3,4,5]$ for the systems have chosen the number of the discrete angular directions as 288 , which is the second largest in the quadrature set. Hence, the efficient numerical scheme of the RTE is still necessary as well as the accuracy of calculation of light propagation inside the heterogeneous reactors.

On the other hand, in the research field of biomedical optics, the RTE has been employed as a forward model of the image reconstruction $[10,11]$. Because biological tissue volumes are highly forward-directed scattering media [12], the same difficulty in the numerical calculation of the RTE arises as in the field of photocatalytic reactions. To overcome the difficulty, the renormalization approaches of the phase function have been developed to satisfy the normalization conditions of the phase function $[13,14,15]$. It has been reported that the renormalization approaches improve the accuracy of the numerical calculation even when using a small number of the discrete angular directions. Hence, the renormalization approaches developed in the field of biomedical optics are expected to be applicable 
for the numerical calculation in the photocatalytic systems. Nevertheless, the application to the photocatalytic systems is not straightforward because the optical properties of the photocatalytic systems in a ultraviolet wavelength range from 290 to $410 \mathrm{~nm}$ are quite different from those of biological tissue volumes in a near infrared wavelength range from 700 to $1100 \mathrm{~nm}$. The anisotropy factor of the aqueous titanium dioxide suspensions strongly depends on the wavelength and in the case of Aldrich, it varies from 0.37 to 0.83 approximately [6], while biological tissue volumes and silica-supported titanium dioxide suspensions are less dependent on the wavelength $[3,16]$. Additionally, in the photocatalytic systems, the scattering coefficient is comparable with the absorption coefficient in a short wavelength range around $290 \mathrm{~nm}$. In biological tissue volumes, the volumetric scattering coefficient ranging from about $100 \mathrm{~cm}^{-1}$ to $1000 \mathrm{~cm}^{-1}$ is much (about two orders of magnitude) larger than the absorption coefficient [17], and the anisotropy factor ranges from about 0.8 to 0.95 showing highly forward-directed scattering. Due to the very large scattering coefficient, the mean free path is very small less than $0.01 \mathrm{~cm}$, and resultantly the highly forward-directed scattering can be approximated to isotropic scattering after many scattering events in the biological tissue volume with the thickness more than $1 \mathrm{~cm}$. Then, several approximations such as the diffusion approximation have been widely employed [18]. However, in the aqueous suspensions of titanium dioxide (Aldrich) with the volumetric scattering coefficient ranging from about $20 \mathrm{~cm}^{-1}$ to $40 \mathrm{~cm}^{-1}$, the mean free path is in the order of $0.05 \mathrm{~cm}$ and the diffusion approximation is not applicable to the system with the thickness less than about $5 \mathrm{~cm}$. Therefore, full calculations of the RTE are necessary without the approximations. These differences in the optical properties suggest the necessity of constructing the accurate and efficient numerical scheme for solving the RTE when the anisotropy of the forward-directed scattering varies and the strength of absorbing is comparable with that of scattering, encountered in photocatalytic systems.

In this paper, we develop numerical schemes for solving the RTE using the renormalization approaches of the phase function with a wide range of anisotropy factor from 0.45 to 0.8 and investigate the influences of forward-directed scattering on the numerical calculation of the RTE. The following section mentions the light propagation model and numerical scheme for solving the RTE using the renormalization approaches. Section 3 provides the numerical results for the normalization conditions of the phase function and numerical solutions of the RTE. Finally, conclusions are mentioned.

\section{Light propagation model}

\subsection{Radiative transfer equation (RTE)}

The RTE for three dimensional media is given [8] in the following formula at low temperatures where light emission can be neglected:

$$
\begin{aligned}
& {\left[\frac{\partial}{v \partial t}+\boldsymbol{\Omega} \cdot \nabla+\mu_{a, \lambda}(\boldsymbol{r})+\mu_{s, \lambda}(\boldsymbol{r})\right] I_{\lambda}(\boldsymbol{r}, \boldsymbol{\Omega}, t)} \\
& =\mu_{s, \lambda}(\boldsymbol{r}) \int_{\mathbb{S}^{2}} d \boldsymbol{\Omega}^{\prime} p\left(\boldsymbol{\Omega}, \boldsymbol{\Omega}^{\prime}\right) I_{\lambda}\left(\boldsymbol{r}, \boldsymbol{\Omega}^{\prime}, t\right)+q(\boldsymbol{r}, \boldsymbol{\Omega}, t) .
\end{aligned}
$$


Here, $I_{\lambda}(\boldsymbol{r}, \boldsymbol{\Omega}, t)$ in $\mathrm{Wcm}^{-2} \mathrm{sr}^{-1}$ represents the light intensity as a function of spatial location $\boldsymbol{r}=(x, y, z) \in \mathbb{R}^{3}$ in cm, angular direction $\Omega=\left(\Omega_{x}, \Omega_{y}, \Omega_{z}\right) \in \mathbb{S}^{2}$ in sr, time $t$ in ps, and $\lambda$ wavelength in nm. $\mu_{a, \lambda}(\boldsymbol{r})$ and $\mu_{s, \lambda}(\boldsymbol{r})$ in $\mathrm{cm}^{-1}$ are the volumetric absorption and scattering coefficients, respectively; $v$ is the speed of light in the medium; $p\left(\boldsymbol{\Omega}, \boldsymbol{\Omega}^{\prime}\right)$ in $\mathrm{sr}^{-1}$ is the phase function with $\boldsymbol{\Omega}^{\prime}$ and $\boldsymbol{\Omega}$ denoting the incident and scattered directions, respectively; and $q(\boldsymbol{r}, \boldsymbol{\Omega}, t)$ in $\mathrm{W} \mathrm{cm}^{-2} \mathrm{sr}^{-1}$ is a source function.

In the research field of photocatalytic reactions, the steady-state RTE has been mostly employed at each individual wavelength because the local volumetric rate of photon absorption (LVRPA) inside the reactor is a fundamental measurement property, defined as

$$
(L V R P A)=\sum_{\lambda \leq \lambda_{b g}} \mu_{a, \lambda}(\boldsymbol{r}) \int_{\mathbb{S}^{2}} d \boldsymbol{\Omega} I(\boldsymbol{r}, \boldsymbol{\Omega})=\sum_{\lambda \leq \lambda_{b g}} \mu_{a, \lambda}(\boldsymbol{r}) \Phi(\boldsymbol{r}) .
$$

Here, $\lambda_{b g}$ represents a wavelength corresponding to the band gap energy and $\Phi(\boldsymbol{r})$ the fluence rate. Because the LVRPA is calculated by a summation over the wavelength, steady-state or frequency-domain measurement of light intensity is useful for receiving light with a wide range of wavelengths. Meanwhile, in the time-resolved measurements of light, it is not easy to reveive light with a broad wavelength range. However, for the determination of the optical properties, timeresolved measurements have the advantage over the steady-state measurement; the time-resolved measurements allow to determine $\mu_{a, \lambda}$ and $\mu_{s, \lambda}$ separately, while the CW measurements cannot [19]. From the discussion above, this study employs the time-dependent RTE. In the following, the subscript, $\lambda$, is omitted for simplicity.

\subsection{Henyey-Greenstein phase function and anisotropy factor}

For the formulation of $p\left(\boldsymbol{\Omega}, \boldsymbol{\Omega}^{\prime}\right)$, the Henyey-Greenstein (HG) phase function [20] is widely employed,

$$
p\left(\boldsymbol{\Omega} \cdot \boldsymbol{\Omega}^{\prime}\right)=\frac{1}{4 \pi} \frac{1-g^{2}}{\left(1+g^{2}-2 g \boldsymbol{\Omega} \cdot \boldsymbol{\Omega}^{\prime}\right)^{3 / 2}} .
$$

The HG phase function assumes that the scattering probability depends on $\boldsymbol{\Omega} \cdot \boldsymbol{\Omega}^{\prime}$ or the polar scattering angle, $\varphi=\cos ^{-1} \boldsymbol{\Omega} \cdot \boldsymbol{\Omega}^{\prime}$. Also, $g \in[-1,1]$ is the anisotropy factor, defined by

$$
g=\frac{\int_{\mathbb{S}^{2}} d \boldsymbol{\Omega}^{\prime} p\left(\boldsymbol{\Omega} \cdot \boldsymbol{\Omega}^{\prime}\right) \boldsymbol{\Omega} \cdot \boldsymbol{\Omega}^{\prime}}{\int_{\mathbb{S}^{2}} d \boldsymbol{\Omega}^{\prime} p\left(\boldsymbol{\Omega} \cdot \boldsymbol{\Omega}^{\prime}\right)}
$$

The $g$-values of silica-supported titanium dioxide and biological tissue volumes are typically larger than $0.8[3,12]$, meaning the highly forward-directed scattering. Meanwhile, the $g$-value of powder titanium dioxide varies from 0.37 to 0.83 [6]. Figure 1 shows the HG phase function (Eq. (3)) as a function of $\varphi$ for the cases of $g=0.0,0.35$, and 0.85 , in logarithmic scale. For isotropic scattering with $g=$ 0.0 , the phase function has a circular shape, i.e., its values are constant over the whole region of $\varphi$. For forward-directed scattering with $g=0.35$, the shape of the phase function deviates from the circular shape, and the values decrease in the backscattering region $(\cos \varphi<0)$ and increase in the forward-scattering region 


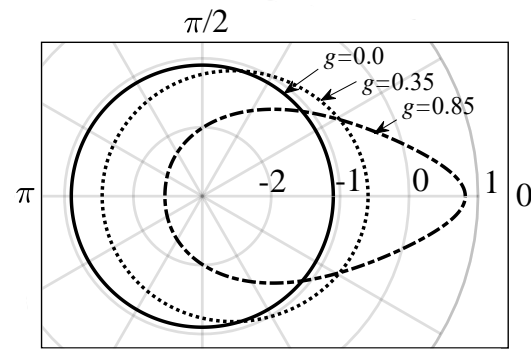

$3 \pi / 2$

Fig. 1 Henyey-Greenstein phase function, $p$, as a function of the polar scattering angle $\varphi$ in polar coordinates and semi logarithmic scale for isotropic scattering $(g=0.0$, solid curve), moderately forward-directed scattering ( $g=0.35$, dotted curve), and highly forward-directed scattering ( $g=0.85$, chain curve). Values of $\varphi$ are denoted outside the frame, and values of $\log _{10} p$ are represented by closed contour lines.

$(\cos \varphi>0)$. When the $g$-value increases, the deviation of the shape from the circle becomes enhanced and the values of the phase function increase exponentially around the forward-scattering region.

Theoretically, the HG phase function satisfies the zeroth and first order normalization conditions at any nonzero values of $g$ as follows:

$$
\begin{aligned}
\int_{\mathbb{S}^{2}} d \boldsymbol{\Omega}^{\prime} p\left(\boldsymbol{\Omega} \cdot \boldsymbol{\Omega}^{\prime}\right) & =1, \\
g^{-1} \int_{\mathbb{S}^{2}} d \boldsymbol{\Omega}^{\prime} p\left(\boldsymbol{\Omega} \cdot \boldsymbol{\Omega}^{\prime}\right) \boldsymbol{\Omega} \cdot \boldsymbol{\Omega}^{\prime} & =1 .
\end{aligned}
$$

\section{Numerical schemes for the RTE}

In this study, the RTE is numerically solved based on the DOM for angular direction and on the finite-difference method for space and time.

\subsection{Discrete ordinates method (DOM)}

The DOM approximates the scattering integral in Eq. (1) to a quadrature sum:

$$
\mu_{s}(\boldsymbol{r}) \int_{\mathbb{S}^{2}} d \boldsymbol{\Omega}^{\prime} p\left(\boldsymbol{\Omega} \cdot \boldsymbol{\Omega}^{\prime}\right) I\left(\boldsymbol{r}, \boldsymbol{\Omega}^{\prime}, t\right) \sim \mu_{s}(\boldsymbol{r}) \sum_{l^{\prime}=1}^{N_{\Omega}} w_{l^{\prime}} p_{l l^{\prime}} I_{l^{\prime}}\left(\boldsymbol{r}, \boldsymbol{\Omega}_{l^{\prime}}, t\right),
$$

where $w_{l^{\prime}}$ is a weight for numerical integration, $\boldsymbol{\Omega}_{l^{\prime}}$ is the $l^{\prime}$-th discrete angular direction, and $N_{\Omega}$ is a total number of the discrete angular directions. In the DOM, a quadrature set of $\left(w_{l}, \Omega_{l}\right)$ needs to be chosen properly because the quadrature set influences the accuracy of the numerical results of the RTE. Among the various kinds of the quadrature sets $[21,22,23]$ developed so far, the most common choice is the level symmetric even (LSE) quadrature set [21] due to its features such as the invariance with respect to 90 -degree axis rotation and line reflection. Also, 
(a)

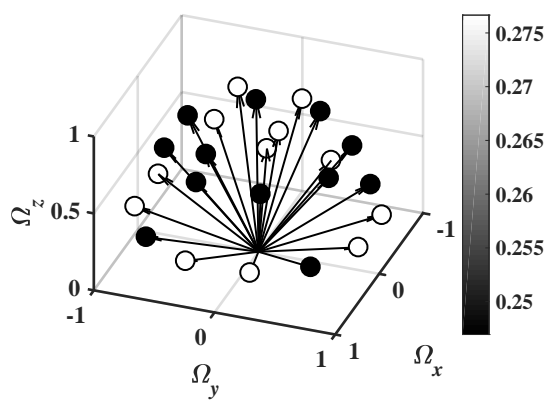

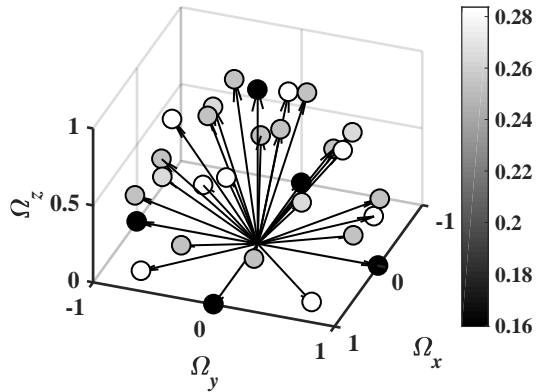

(b)

Fig. 2 Discrete angular directions and weights, $\Omega_{l}$ and $w_{l}$, in the half-sphere $z \geq 0$ for (a) the LSE set with $N_{\Omega}=48$ and (b) Lebedev set with $N_{\Omega}=50$.

the Lebedev quadrature set $[24,25]$ has been reevaluated because the set is as accurate as the LSE set $[26,27,14]$. Figure 2 shows examples of the distributions of $\left(w_{l}, \Omega_{l}\right)$ for the LSE set with $N_{\Omega}=48$ and for the Lebedev set with $N_{\Omega}=50$, respectively, in the half-sphere $z \geq 0$. Here, the arrow and grayscale color of circle denote $\Omega_{l}$ and $w_{l}$, respectively; and the values are calculated by referring to the original papers $[21,24,25]$. As shown in Fig. 2, the distribution of $\left(w_{l}, \boldsymbol{\Omega}_{l}\right)$ for the LSE set is different from that of the Lebedev set even though the values of $N_{\Omega}$ are almost the same to each other.

The previous studies focusing on light propagation in photocatalytic reactors have chosen the LSE set with $N_{\Omega}=288$ to obtain the accurate results of the RTE $[3,4,5]$. However, the value of $N_{\Omega}=288$ leads to high computational costs. To reduce the computational costs, hence, we choose the LSE set with $N_{\Omega}=48$ and the Lebedev set with $N_{\Omega}=50$. In general, the matrix size in the numerical calculations is proportional to $\left(N_{\Omega}\right)^{2}$, so that the case of $N_{\Omega}=50$ reduces the matrix size to approximately $3 \%$ of that in the case of $N_{\Omega}=288$. In addition, for improvement of the accuracy, we employ the renormalization approaches of the phase function, explained in the next subsection.

\subsection{Renormalization approaches of the phase function}

In highly forward-directed scattering, the numerical calculations of the RTE require a large value of $N_{\Omega}$ to obtain accurate results. As shown in Fig. 1, the values of the phase function are strongly dependent on $\varphi$ in highly forward-directed scattering, and the $\varphi$-dependence results in unsatisfaction of the normalization conditions (Eqs. (5) and (6)) in the numerical calculations based on the DOM at small value of $N_{\Omega}$.

To obtain the accurate results of the RTE at a small value of $N_{\Omega}$, we employ the renormalization approaches in the zeroth and first orders. Among the zeroth order renormalization approaches, we employ the Liu and co-worker's formula [13] in this paper because of its simple form. In their approach, the phase function is renormalized to satisfy the zeroth order normalization condition (Eq. (5)):

$$
\hat{p}_{l l^{\prime}}=f_{l} p_{l l^{\prime}}, \quad f_{l}=\left[\sum_{l^{\prime}=1}^{N_{\Omega}} w_{l^{\prime}} p_{l l^{\prime}}\right]^{-1},
$$


where $\hat{p}_{l l^{\prime}}$ denotes the renormalized phase function in the discrete form and $f_{l}$ a renormalizing factor. It has been reported that the zeroth order renormalization approach provides accurate results of the RTE at a small value of $N_{\Omega}$ comparing with the results without the renormalization approach.

We have recently proposed the first order renormalization approach of the phase function to satisfy the zeroth and first order normalization conditions (Eqs. (5) and (6)) [15]. In the proposed approach, $\hat{p}_{l l^{\prime}}$ is given as

$$
\hat{p}_{l l^{\prime}}=f_{l} W_{l l^{\prime}} p_{l l^{\prime}}, \quad f_{l}=\left[\sum_{l^{\prime}=1}^{N_{\Omega}} w_{l^{\prime}} W_{l l^{\prime}} p_{l l^{\prime}}\right]^{-1},
$$

where $W_{l l^{\prime}}$ denotes a renormalizing matrix calculated by the double exponential formula $[28,29]$. The details are referred to [15].

We have confirmed that the first order renormalization approach can improve the accuracy of the numerical results of the RTE comparing with the zeroth order renormalization approach, but only in the case of the optical properties: $g=0.9$, $\mu_{s}=140 \mathrm{~cm}^{-1}$, and $\mu_{a}=0.38 \mathrm{~cm}^{-1}$. Hence, in this paper, the verification of the proposed approach is tested in a wide range of $g$-values and low values of $\mu_{s}$ comparable with those of $\mu_{a}$. Although the verification tests of the proposed approach for various kinds of quadrature sets and a wide range of $N_{\Omega}$ are significant, the tests will be discussed elsewhere.

\subsection{Finite difference method}

We employ the 3rd order weighted essentially non oscillatory scheme [30, 31] for spatial discretization and the 3rd order total variation diminishing-Runge-Kutta method [32] for temporal discretization, respectively. For the details, refer to [33]. In the finite difference method, $x, y, z$, and $t$ are discretized as $x_{i}=i \Delta x(i \in$ $\left.\left\{0, \cdots, N_{x}\right\}\right), y_{j}=j \Delta y\left(j \in\left\{0, \cdots, N_{y}\right\}\right), z_{k}=k \Delta z\left(k \in\left\{0, \cdots, N_{z}\right\}\right)$, and $t_{m}=m \Delta t\left(m \in\left\{0, \cdots, N_{t}\right\}\right)$ with the constant step sizes of $\Delta x, \Delta y, \Delta z$, and $\Delta t$, respectively, and the numbers of the grid nodes and timesteps of $N_{x}$, $N_{y}, N_{z}$, and $N_{t}$, respectively. At the boundary of a medium, the refractive-index mismatched boundary condition is employed.

The source code for the numerical calculation was written in the $\mathrm{C}$ programming language, and all the matrices were compressed to vectors as the compressed row storage format. Also, parallel CPU programming was implemented with 48 thread computers (Intel Xeon E5-2690v3@ 2GHz) by using the OpenMP, which is a portable and shared-memory programming scheme.

3.4 Numerical phantoms modeling the aqueous titanium dioxide suspensions for Aldrich powder and numerical conditions

In this paper, we consider numerical phantoms modeling the aqueous titanium dioxide suspensions for Aldrich powder by referring to [6]. In the reference, values of the specific absorption and scattering coefficients, $\mu_{a}^{*}$ and $\mu_{s}^{*}$, in $\mathrm{cm}^{2} \mathrm{~g}^{-1}$; and $g$ are determined in the ultraviolet wavelength range. From the results, we obtain the values of $\mu_{a}^{*}, \mu_{s}^{*}$, and $g$ at a step of 0.5 of the $g$-values in a range of the $g$-values 
Table 1 Optical properties of the aqueous suspensions of titanium dioxide (Aldrich) [6]. Volumetric absorption and scattering coefficients, $\mu_{a}$ and $\mu_{s}$, are given by $C_{m} \mu_{a}^{*}$ and $C_{m} \mu_{s}^{*}$ with the catalyst concentration of $C_{m}=10^{-3}\left[\mathrm{~g} \mathrm{~cm}^{-3}\right]$; and the specific absorption and scattering coefficients, $\mu_{a}^{*}$ and $\mu_{s}^{*}$, respectively. Conditions for the numerical calculations of the RTE: spatial step size, $\Delta x$, and source-detector distance, $\rho$.

\begin{tabular}{ccccc||cc}
\hline$g[-]$ & $\mu_{a}^{*}\left[\mathrm{~cm}^{2} \mathrm{~g}^{-1}\right]$ & $\mu_{s}^{*}\left[\mathrm{~cm}^{2} \mathrm{~g}^{-1}\right]$ & $\mu_{a}\left[\mathrm{~cm}^{-1}\right]$ & $\mu_{s}\left[\mathrm{~cm}^{-1}\right]$ & $\Delta x[\mathrm{~cm}]$ & $\rho[\mathrm{cm}]$ \\
\hline 0.80 & $1.79 \times 10^{4}$ & $1.91 \times 10^{4}$ & $1.79 \times 10$ & $1.91 \times 10$ & $2.50 \times 10^{-2}$ & 1.50 \\
0.75 & $1.67 \times 10^{4}$ & $2.12 \times 10^{4}$ & $1.67 \times 10$ & $2.12 \times 10$ & $2.50 \times 10^{-2}$ & 1.50 \\
0.70 & $1.50 \times 10^{4}$ & $2.35 \times 10^{4}$ & $1.50 \times 10$ & $2.35 \times 10$ & $1.60 \times 10^{-2}$ & 1.20 \\
0.65 & $1.30 \times 10^{4}$ & $2.60 \times 10^{4}$ & $1.30 \times 10$ & $2.60 \times 10$ & $1.50 \times 10^{-2}$ & 0.99 \\
0.60 & $1.08 \times 10^{4}$ & $2.87 \times 10^{4}$ & $1.08 \times 10$ & $2.87 \times 10$ & $1.25 \times 10^{-2}$ & 0.90 \\
0.55 & $8.19 \times 10^{3}$ & $3.17 \times 10^{4}$ & 8.19 & $3.17 \times 10$ & $1.25 \times 10^{-2}$ & 0.85 \\
0.50 & $5.30 \times 10^{3}$ & $3.49 \times 10^{4}$ & 5.30 & $3.49 \times 10$ & $1.25 \times 10^{-2}$ & 0.75 \\
0.45 & $2.68 \times 10^{3}$ & $3.79 \times 10^{4}$ & 2.68 & $3.79 \times 10$ & $1.25 \times 10^{-2}$ & 0.70 \\
\hline
\end{tabular}

from 0.45 to 0.8 as listed in Table 1 . Then, by setting the catalyst concentration of $C_{m}$ as $10^{-3} \mathrm{~g} \mathrm{~cm}^{-3}$, and by using the relations of $\mu_{a}=C_{m} \mu_{a}^{*}$ and $\mu_{s}=C_{m} \mu_{s}^{*}$, we obtain the values of $\mu_{a}$ and $\mu_{s}$. Table 1 shows that at $g=0.8$, the values of $\mu_{a}$ and $\mu_{s}$ are in the same order, while as the $g$-value decreases, the $\mu_{a}$-value decreases and the $\mu_{s}$-value increases. These tendencies are different from those of biological tissue volumes.

We consider a homogeneous cubic medium with a side of $3.1 \mathrm{~cm}$ as a first step toward the numerical calculations of the RTE in heterogeneous media, because the analytical solutions of the RTE in infinite homogeneous media are applicable for the verification tests of the numerical calculations. Extensions of the developed numerical schemes into the heterogeneous media are straightforward. The source and detector are located inside the medium at $\boldsymbol{r}_{s}=(1.55 \mathrm{~cm}, 1.55 \mathrm{~cm}, 0.93 \mathrm{~cm})$ and $\boldsymbol{r}_{d}=\boldsymbol{r}_{s}+\rho \hat{\boldsymbol{e}}_{z}$ with the source-detector distance of $\rho$ and the unit vector of $z$-axis of $\hat{\boldsymbol{e}}_{z}$ to suppress the boundary effects because we employ the analytical solution of the RTE for infinite media.

Table 1 also lists the values of $\Delta x$ and $\rho$ at each value of $g$, where we set $\Delta x=\Delta y=\Delta z$. The appropriate values of $\Delta x$ depend on the optical properties; as the values of $\mu_{a}+\mu_{s}$ are larger, the values of $\Delta x$ are smaller. The step sizes are properly determined so that the numerical results of the RTE are unchanged even as the step sizes are finer than the determined values. Because the numerical calculation of the RTE treats multiple scattering events, we set the values of $\rho$ so that the values of $\rho \mu_{s}$ are constant at approximately 27.

\subsection{Analytical solution of the RTE}

In this paper, we employ the analytical solution of the RTE for the time-domain fluence rate, $\Phi(\boldsymbol{r}, t)$, in homogeneous infinite media to evaluate the numerical errors of the RTE calculations using the developed schemes here. Recently, the analytical solution of the RTE with anisotropy scattering $(g \neq 0)$ in time domain has been derived using a single-sided Laplace transformation of the spherical harmonics expansions [34]. In the reference, the analytical form of the fluence rate, $\Phi_{\text {ana }}(r, t)$, 
is given as

$$
\Phi_{\text {ana }}(r, t)=\frac{1}{2 r R^{2}} \sum_{\kappa=1}^{\infty} \kappa I_{\kappa 0}(t) \sin \left(\xi_{\kappa 0} r\right),
$$

where $r=|\boldsymbol{r}|, R$ represents the radius of a large sphere, $\kappa$ discrete wavenumber, $I_{\kappa 0}(t)$ the time-dependent mode of the intensity, $\xi_{\kappa 0}$ the positive root of the equation of $j_{0}\left(\xi_{\kappa 0} R\right)=0$ with $j_{0}$ being the zeroth order spherical Bessel function of the first kind. Independent study [19] has verified the analytical solution (Eq. (10)) by comparing with Monte Carlo simulations and experimental data. Because Eq. (10) forms the summation over $\kappa$, numerical calculation is partially necessary. Hence, we employ an open source MATLAB code [34] for the calculation of Eq. (10).

\section{Numerical results}

4.1 First order normalization condition of the phase function

In this subsection, we investigate numerical errors of the normalization conditions of the phase function (Eqs. (5) and (6)) in a wide range of the $g$-values from 0.35 to 0.95 . At each value of $g$, the numerical errors are calculated for the five combinations of the quadrature set and renormalization approaches: (i) the LSE set with $N_{\Omega}=48$ using the zeroth order approach (Eq. (8)); (ii) the Lebedev set with $N_{\Omega}=50$ using the zeroth order approach; (iii) the LSE set with $N_{\Omega}=48$ using the first order approach (Eq. (9)); (iv) the Lebedev set with $N_{\Omega}=50$ using the first order approach; and (v) the LSE set with $N_{\Omega}=288$ using the zeroth order approach.

We have confirmed that the numerical errors of the zeroth order normalization condition (Eq. (5)) are zero in all the cases of $g$-values for the five combinations, so that we discuss here the numerical errors of the first order normalization condition (Eq. (6)). We consider numerical integration, $S_{1}^{l}$ :

$$
S_{1}^{l}=g^{-1} \sum_{l^{\prime}=1}^{N_{\Omega}} w_{l^{\prime}} \hat{p}_{l l^{\prime}} \boldsymbol{\Omega}_{l} \cdot \boldsymbol{\Omega}_{l^{\prime}}, \quad l=1,2, \cdots, N_{\Omega} .
$$

The ideal value of $S_{1}^{l}$ is unity. From the calculated values of $S_{1}^{l}$, we evaluate the mean absolute percentage error, $e_{1}$ :

$$
e_{1}=N_{\Omega}^{-1} \sum_{l=1}^{N_{\Omega}}\left|S_{1}^{l}-1\right| \times 100 .
$$

Figure 3 shows the numerical errors, $e_{1}$, for the five combinations at different $g$ values from 0.35 to 0.95 . In Combinations (i) and (ii), the errors tend to increase up to $10 \%$ as the $g$-value increases. This is because as the $g$-value increases, the phase function has a sharper peak and the $\varphi$-dependence of the phase function is enhanced, but the zeroth order approach renormalizes the phase function by a constant value independent of $\varphi$. Meanwhile, the errors in Combinations (iii) and (iv) are smaller less than $1 \%$ in a whole region of the $g$-values. This is attributed to the fact that the first order approach renormalizes the phase function by the weight matrix, $W_{l l^{\prime}}$, dependent of $\varphi$. The results for Combinations (i) to (iv) 


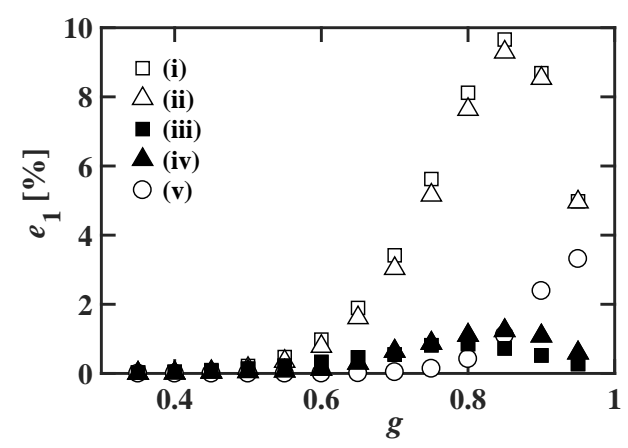

Fig. 3 Mean absolute percentage errors, $e_{1}$, of $S_{1}^{l}$ (Eq. (12)) as a function of the $g$-values for the five combinations of the quadrature sets and renormalization approaches. Open and solid symbols represent the results using the zeroth order and first order renormalization approaches, respectively. Squares represent those for the LSE set with $N_{\Omega}=48$; triangles those for the Lebedev set $N_{\Omega}=50$; and circles those for the LSE set with $N_{\Omega}=288$.

suggest that the first order renormalization approach plays a significant role of the error reductions especially at the large $g$-values more than 0.6.

The numerical errors for Combination (v) corresponding to the large value of $N_{\Omega}$ are compared with those at the small values of $N_{\Omega}$ in Fig. 3. Here, it is noted that the previous researches for the photocatalytic reactors $[3,4,5]$ adopted the same value of $N_{\Omega}=288$ as Combination (v), but did not employed the renormalization approach. Focusing on the results for the zeroth order approach, the errors for Combination (v) are smaller than those for Combinations (i) and (ii) at the small values of $g$ less than 0.6 , but increase up to $4 \%$ as the $g$-value reaches at 0.95. Also, the results for Combinations (iii) to (v) show that at the large $g$-values more than 0.8 , the errors in the case of the first order approach are smaller than those of the zeroth order approach, even though the use of $N_{\Omega}=288$ results in high computational costs compared with that of $N_{\Omega}=48$.

Our previous paper [15] has suggested that the values of $e_{1}$ are positively correlated to the numerical errors of the RTE at $g=0.9$. Also, the paper has found out that an acceptable value of $e_{1}$ is approximately $1 \%$, below which the numerical results of the RTE are little changed and accurately agree with the analytical solutions of the RTE. As shown in Fig. 3, the values of $e_{1}$ in the case of the first order approach are less than approximately $1 \%$ in the whole range of the $g$-values, so that the results indicate that the numerical results of the RTE using the first order approach would be accurate in the range of the $g$-values.

\subsection{Fluence rate}

In this subsection, we investigate the numerical errors of the RTE calculations using the developed schemes for the numerical phantoms modeling the titanium dioxide suspensions as listed in Table 1 . We calculated the temporal profiles of the fluence rate, $\Phi(\boldsymbol{r}, t)=\int_{\mathbb{S}^{2}} d \boldsymbol{\Omega} I(\boldsymbol{r}, \boldsymbol{\Omega}, t)$, for the numerical phantoms and compared them with the analytical solution of the RTE (Eq. (10)) for infinite homogeneous media. As examples, Fig. 4 shows the numerical results of $\Phi(\boldsymbol{r}, t)$ at $g=0.45$ and 0.8 for Combinations (i) and (iii); and the analytical solution. As shown in 

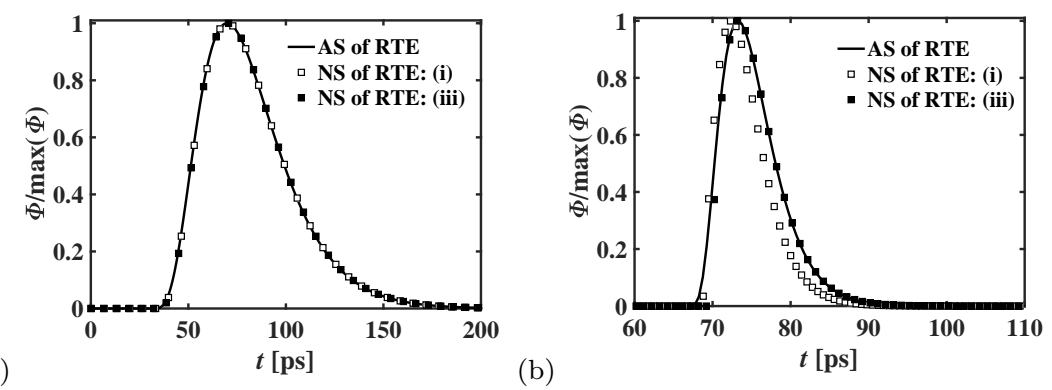

Fig. 4 Temporal profiles of the fluence rate, $\Phi$, in the numerical phantoms modeling the titanium dioxide suspensions as listed at Table 1: (a) $g=0.45$ and (b) $g=0.80$, respectively. Open and solid squares represent the numerical solutions (NS) of the RTE for Combinations (i) and (iii), respectively; and solid lines the analytical solutions (AS) of the RTE (Eq. (10)).

Fig. 4(a), the temporal profiles have relatively broad peaks (at FWHM of about 50 ps) due to the condition of the scattering dominance over the absorption as listed in Table 1. The numerical results using the both renormalization approaches nicely agree with the analytical solution at the small $g$-value of 0.45 . These results are probably because the values of $e_{1}$ are sufficiently small at $g=0.45$ as shown in Fig. 3. As shown in Fig. 4(b), the temporal profiles at $g=0.8$ have sharper peaks (at FWHM of about $10 \mathrm{ps)}$ than those at $g=0.45$ due to the conditions of the scattering comparable with the absorption in addition to the large value of $g$. Generally, such a condition requires a small value of $\Delta x$ and large value of $N_{\Omega}$ to attain the accurate results of the numerical calculations, resulting in much higher computational costs. Despite the use of the small value of $N_{\Omega}$, the numerical result of $\Phi(\boldsymbol{r}, t)$ using the first order approach agrees with the analytical solution due to the small value of $e_{1}$. Meanwhile, the result using the zeroth order approach largely deviates from the analytical solution because of the large value of $e_{1}$.

Next, we investigate the difference in the shape of the temporal profiles of the fluence rate normalized by their peak values, $\hat{\Phi}$, between the numerical and analytical solutions of the RTE, without discussion of their magnitudes. The difference in $\hat{\Phi}$ between the numerical and analytical solutions of the RTE was evaluated by the mean absolute percentage error, $e_{\Phi}$ :

$$
e_{\Phi}=\frac{1}{M_{2}-M_{1}} \sum_{m=M_{1}}^{M_{2}}\left|\frac{\hat{\Phi}^{m}-\hat{\Phi}_{a n a}\left(t_{m}\right)}{\hat{\Phi}_{\text {ana }}\left(t_{m}\right)}\right| \times 100,
$$

where $\hat{\Phi}^{m}$ and $\hat{\Phi}_{\text {ana }}\left(t_{m}\right)$ represent the numerical and analytical solutions for $\hat{\Phi}$ at the $m$-th time step, $t_{m}$, respectively; and the summation with respect to $m$ is over the time period from the time of $t_{M_{1}}$ when $\hat{\Phi}_{\text {ana }}$ reaches the peak, to the time of $t_{M_{2}}$ when $\hat{\Phi}_{\text {ana }}$ falls to $10^{-1.5} \simeq 0.032$ after the peak. Here, the time period earlier than $t_{M_{1}}$ is excluded from the calculation of $e_{\Phi}$ because the analytical solution (Eq. (10)) oscillates in the early time period due to the spherical harmonics expansion, and because in the numerical solution the rise of the temporal profile from zero is less sharp than in the analytical solution.

At each value of $g$, we calculate $e_{\Phi}$ for the four combinations of the quadrature set and renormalization approaches: Combinations (i) to (iv) defined in the previous subsection. The reasons to exclude Combination (v) are that the accuracy of 


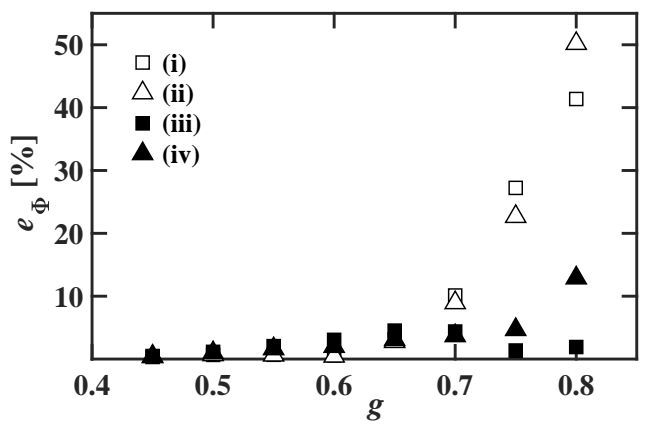

Fig. 5 The difference in $\hat{\Phi}, e_{\Phi}$, between the numerical and analytical solutions of the RTE with different $g$-values for Combinations (i) to (iv). Other details are the same as in Fig. 3.

the numerical results of the RTE using this combination has been already shown in the previous studies (e.g. [35]); and that one of our purposes is to develop efficient and accurate numerical schemes of the RTE with the small values of $N_{\Omega}$.

Figure 5 shows the errors of $\hat{\Phi}, e_{\Phi}$ as a function of the $g$-values for the four combinations. At the small $g$-values less than 0.7 , the both renormalization approaches provide almost the same results for $e_{\Phi}$, while at the large $g$-values larger than 0.7 , the first order approach can reduce the numerical errors comparing with the zeroth order approach. The results suggest that the positive correlation between $e_{1}$ and $e_{\Phi}$ would hold for the different $g$-values. The positive correlation probably comes from the error accumulation of the phase function to the numerical results of the RTE during multiple scattering events.

\section{Conclusions}

We developed the numerical schemes of the DOM-based RTE for numerical phantoms modeling aqueous titanium dioxide suspensions, in which the anisotropy of the forward-directed scattering varies and the strength of absorption is comparable to that of scattering. Then, we employ the quadrature sets with the small values of $N_{\Omega}$ for improvement of the efficiency of the numerical calculation, and the two kinds of renormalization approaches for improvement of the accuracy of the numerical calculation. We found out that at the small $g$-values less than approximately 0.7 , the both approaches can provide the accurate results of the RTE due to the reduction of the errors of the phase function. At the large $g$-values more than 0.7 , the first order approach still provides the accurate results, while the zeroth order approach does not due to the large errors of the phase function. Hence, the numerical scheme of the RTE with the small value of $N_{\Omega}$ using the first order approach has a possibility of the accurate and efficient calculations of light propagation inside the photocatalytic reactors.

Acknowledgements This work was partially supported by JSPS KAKENHI Grant Numbers 15K17980, 15K06125, and 16H02155. The first author learned the research topic of photocatalyst at the Mathematics in (bio)Chemical Kinetics and Engineering (MaCKiE) 2017 conference held in Budapest, Hungary, in 2017. We wish to express appreciation to Mr. K. Tabayashi, Mr. S. Endo, and Mr. K. Nomura for fruitful discussion. 


\section{References}

1. Machalický, O., Lichý, L., Tomica, M., Hrdina, R., Šolcová, O.: Photocatalysis of sulfo and carboxy derivatives of indigo in a TiO2 slurry with the use of polychromatic irradiation. Reac. Kinet. Mech. Cat. 107(1) (2012) 63-77

2. Grčić, I., Vujević, D., Žižek, K., Koprivanac, N.: Treatment of organic pollutants in water using $\mathrm{TiO} 2$ powders: photocatalysis versus sonocatalysis. Reac. Kinet. Mech. Cat. 109(2) (2013) 335-354

3. Maruga, J., Grieken, R.V., Alfano, O.M., Cassano, A.E.: Optical and Physicochemical Properties of Silica-Supported TiO2 Photocatalysts. AIChE J. 52(8) (2006) 2832-2843

4. Maruga, J., Grieken, R.V., Cassano, A.E., Alfano, O.M.: Quantum efficiency of cyanide photooxidation with $\mathrm{TiO} 2 / \mathrm{SiO} 2$ catalysts : Multivariate analysis by experimental design. Catal. Today 129 (2007) 143-151

5. Maruga, J., Grieken, R.V., Cassano, A.E., Alfano, O.M.: Intrinsic kinetic modeling with explicit radiation absorption effects of the photocatalytic oxidation of cyanide with $\mathrm{TiO} 2$ and silica-supported TiO2 suspensions. Appl. Catal. B: Environ. 85 (2008) 48-60

6. Satuf, M.L., Brandi, R.J., Cassano, A.E., Alfano, O.M.: Experimental Method to Evaluate the Optical Properties of Aqueous. Ind. Eng. Chem. Res. 44 (2005) 6643-6649

7. Ortiz, G.B., la Plata, D., Alfano, O.M., Cassano, A.E.: Optical properties of goethite catalyst for heterogeneous photo-Fenton reactions Comparison with a titanium dioxide catalyst. Chem. Eng. J. 137 (2008) 396-410

8. Chandrasekhar, S.: Radiative Transfer. Dover, New York (1960)

9. Carlson, B.G., Lee, C.E.: Mechanical Quadrature and the Transport Equation. Los Alamos Scientific Laboratory Report 2573 (1961)

10. Gibson, A.P., Hebden, J.C., Arridge, S.R.: Recent advances in diffuse optical imaging. Phys. Med. Biol. 50(4) (2005) R1-R43

11. Yamada, Y., Okawa, S.: Diffuse Optical Tomography : Present Status and Its Future. Opt. Rev. 21(3) (2014) 185-205

12. Cheong, W.F., Prahl, S.A., Welch, A.J.: A review of the optical properties of biological tissue. IEEE J. Quantum Electron 26(12) (1990) 2166-2185

13. Liu, L., Ruan, L., Tan, H.: On the discrete ordinates method for radiative heat transfer in anisotropically scattering media. Int. J. Heat Mass Transfer 45(15) (2002) 3259-3262

14. Long, F., Li, F., Intes, X., Kotha, S.P.: Radiative transfer equation modeling by streamline diffusion modified continuous Galerkin method. J. Biomed. Opt. 21(3) (2016) 036003

15. Fujii, H., Okawa, S., Yamada, Y., Hoshi, Y., Watanabe, M.: Renormalization of the highly forward-peaked phase function using the double exponential formula for radiative transfer. J. Math. Chem. 54(10) (2016) 2048-2061

16. Jacques, S.L.: Optical properties of biological tissues : a review. Phys. Med. Biol. 58 (2013) R37-R61

17. Bashkatov, A.N., Genina, E.A., Tuchin, V.V.: OPTICAL PROPERTIES OF SKIN, SUBCUTANEOUS, AND MUSCLE TISSUES : A REVIEW. J. Innov. Opt. Health Sci. 4(1) (2011) 9-38

18. Okawa, S., Hoshi, Y., Yamada, Y.: Improvement of image quality of timedomain diffuse optical tomography with lp sparsity regularization. Biomed. 
Opt. Express 2(12) (2011) 3334-3348

19. Kamran, F., Abildgaard, O.H.A., Subash, A.A., Andersen, P.E., Anderssonengels, S., Khoptyar, D.: Computationally effective solution of the inverse problem in time-of-flight spectroscopy. Opt. Express 23(5) (2015) 6937-6945

20. Henyey, L.G., Greenstein, L.J.: Diffuse radiation in the galaxy. J. Astrophys. 93 (1941) 70-83

21. Fiveland, W.A.: The selection of discrete ordinate quadrature sets for anisotropic scattering. ASME, HTD-vol. 160, Fundamentals of radiation heat transfer (1991) 89-96

22. Carlson, B.G.: Quadrature Tables of Equal Weight EQn Over the Unit sphere. Los Alamos Scientific Laboratory Report 4734 (1971)

23. Balsara, D.: Fast and accurate discrete ordinates methods for multidimensional radiative transfer. Part I, basic methods. J. Quant. Spectrosc. Radiat. Transfer 69 (2001) 671-707

24. Lebedev, V.I.: Values of the nodes and weights of ninth to seventeenth order gauss-markov quadrature formulae invariant under the octahedron group with inversion. USSR Comput. Math. Math. Phys. 15(1) (1975) 44-51

25. Lebedev, V.I.: Spherical quadrature formulas exact to orders 25-29. Siberian Math. J. 18(1) (1977) 99-107

26. Gregersen, B.A., York, D.M.: High-order discretization schemes for biochemical applications of boundary element solvation and variational electrostatic projection methods. J. Chem. Phys. 122(19) (2005) 194110

27. Sanchez, R.: Prospects in deterministic three-dimensional whole-core transport calculations. Nucl. Eng. Technol. 44(5) (2012) 113-150

28. Takahasi, H., Mori, M.: Double exponential formulas for numerical indefinite integration. Publ. RIMS Kyoto Univ. 9 (1974) 721-741

29. Mori, M., Sugihara, M.: The double-exponential transformation in numerical analysis. J. Comput. Appl. Math. 127 (2001) 287-296

30. Jiang, G.S., Shu, C.W.: Efficient Implementation of Weighted ENO Schemes. J. Comput. Phys. 126(1) (1996) 202-228

31. Henrick, A.K., Aslam, T.D., Powers, J.M.: Mapped weighted essentially nonoscillatory schemes: Achieving optimal order near critical points. J. Comput. Phys. 207(2) (2005) 542-567

32. Gottlieb, S., Shu, C.W.: Total variation diminishing Runge-Kutta schemes. Math. Comp. 67(221) (1998) 73-85

33. Fujii, H., Yamada, Y., Kobayashi, K., Watanabe, M., Hoshi, Y.: Modeling of light propagation in the human neck for diagnoses of thyroid cancers by diffuse optical tomography. Int. J. Numer. Meth. Biomed. Engng. 33(e2826) (2017) $1-12$

34. Liemert, A., Kienle, A.: Infinite space Green's function of the time-dependent radiative transfer equation. Biomed. Opt. Express 3(3) (2012) 543

35. Klose, A.D., Larsen, E.W.: Light transport in biological tissue based on the simplified spherical harmonics equations. J. Comput. Phys. 220 (2006) 441470 